\title{
Análisis de indicadores macro y microscópicos para establecer el periodo de máxima intensidad de desove de la anchoveta Engraulis ringens en la zona norte de Chile
}

Analysis of macro and microscopic indicators to establish the period of maximum intensity of spawning of anchovy Engraulis ringens of northern Chile

\author{
Carola Hernández-Santoro ${ }^{1}$, Graciela Pérez-Mora², \\ Eduardo Díaz-Ramos² y Gabriela Böhm-Stoffel ${ }^{1}$
}

\begin{abstract}
'Instituto de Fomento Pesquero, IFOP, Blanco 839, Valparaíso, Chile. carola.hernandez@ifop.cl ${ }^{2}$ Instituto de Fomento Pesquero, IFOP, Avda. Arturo Prat Sitio 3, Iquique, Chile

Abstract.- Although the period of maximum reproductive activity of anchoveta has long been established between July and December, this period can be further defined using microscopical maturity information. This rather unknown biological information is relevant to specify the period of maximum spawning intensity for the management of the fishery. In this work the period of anchoveta maximum spawning intensity is defined using macro and microscopic sexual maturity data from the years 2000 to 2010. The monthly variability of the Gonadosomatic Index (IGS) showed a higher reproductive activity from July onwards reaching a maximum value in August, with high IGS values been observed until December. Microscopical analysis allowed to precise this period between the first half of July and mid October. On the basis of expert judgment, the onset of maximum spawning intensity of anchovy can be established when the Spawning Activity Index (IAD) attains a sustained value of at least $25 \%$ during over 2 weeks non necessarily in a row, with a IGS equal or higher than $6 \%$.
\end{abstract}

Key words: Engraulis ringens, gonadosomatic index, spawning period, reproductive activity, northern Chile

Resumen.- El período de máxima actividad reproductiva de la anchoveta en la zona norte ha sido establecido de julio a diciembre, siendo posible precisarlo mediante el uso de técnicas microscópicas. Al respecto, este análisis es menos utilizado por su alto costo no obstante es relevante para precisar el período de máxima intensidad desove (MID) para el manejo de la pesquería. En este trabajo se determinó el periodo de máxima intensidad desove en base a la información macro y microscópica de la gónada entre el 2000 y 2010. La evolución mensual del Indice Gonadosomático (IGS) evidenció una mayor actividad reproductiva a partir de julio con un máximo en agosto y altos índices hasta diciembre, período que se precisó con el análisis histológico el que se extiende entre la primera quincena de julio y mediados de octubre. El período de máxima intensidad de desove de la anchoveta según criterio experto se definió cuando se mantiene por sobre 2 semanas no necesariamente consecutivas un valor del índice de actividad de desove (IAD) igual o superior a $25 \%$ con IGS de $6 \%$ o superiores.

Palabras clave: Engraulis ringens, índice gonadosomático, periodo de desove, actividad reproductiva, norte de Chile

\section{INTRODUCCIÓN}

En la zona norte de Chile, la pesquería de pequeños pelágicos, ha presentado 3 períodos: el primero de 1955 a 1976, basado casi exclusivamente en la extracción de anchoveta (Engraulis ringens), donde se observó un crecimiento sostenido del recurso hasta 1966 alcanzando $1,1$ millones de toneladas ( $t)$, para luego registrar una fuerte reducción de las capturas (promedio 1967-1972 de 540 mil t), siendo más notable posterior al evento El Niño 1972-1973, con niveles promedio de 330 mil t (1974-1976). El segundo período comienza a mediados de la década del 70 y corresponde a una etapa de bajas capturas de anchoveta y un aumento notable en la abundancia de sardina española (Sardinops sagax), especie que sostuvo la pesquería hasta fines de la década del 80 con capturas promedio de 1,8 millón de $t$ entre 1978 y 1989. El tercer periodo se establece desde mediados de los 80 y se caracteriza por una mayor abundancia y estabilización en las capturas, las que se nivelan en torno a los 1,3 millones de t, cifra que fue disminuyendo para promediar las 783 mil t en el período 2006-2010. Este comportamiento indica que en los últimos 50 años se evidencian fuertes fluctuaciones a diferentes escalas de tiempo en la población de anchoveta, cambios que se asocian a la variabilidad interanual del régimen ambiental y que son considerados como importantes factores moduladores en los procesos biológicos y reproductivos de la anchoveta 
(Jordan 1976, Claramunt et al. 1997, Buitrón \& Perea 2000, Castro et al. 2000, Cubillos \& Claramunt 2009, Chávez et al. 2009, Purca et al. 2010, Mori et al. 2011). La anchoveta es considerada una especie pelágica-costera y desovante parcial, lo que implica que en un determinado período de tiempo es posible encontrar ejemplares en diferentes estados de madurez sexual (Arancibia et al. 1994). No obstante, el período de desove principal se distingue de agosto a marzo (Santander et al. 1984), con una mayor intensidad en septiembre, durante el invierno austral (Einarsson et al. 1966) y con un segundo máximo registrado durante febrero-marzo correspondiente al verano austral (Csirke et al. 1996). Si bien existen diversos estudios enfocados en el conocimiento de la variabilidad y la estacionalidad del potencial reproductivo de las hembras de E. ringens (Einarsson et al. 1966, Jordan 1976, Claramunt et al. 2007), existe una incertidumbre en cuánto a la variabilidad interanual. Esta variabilidad se aprecia tanto en la duración como en el inicio y fin de la actividad reproductiva, producto de la influencia que tienen los cambios ambientales en su proceso biológico (desove y reclutamiento). Debido a la importancia económica que tiene el recurso y considerando la dinámica oceanográfica que presenta la zona la norte de Chile, se analizó mediante el uso de indicadores macroscópicos como el índice gonadosomático (IGS) y proporción de hembras maduras y microscópicos como el índice de actividad de desove (IAD) y el índice de atresia ovárica (IAO), el período de desove, permitiendo identificar con una mayor precisión el período de máxima intensidad de desove (MID) de la anchoveta para la zona norte.

\section{Materiales Y MÉTODOS}

El área de estudio corresponde a la zona norte de Chile $\left(18^{\circ} 21^{\prime}\right.$ y $\left.24^{\circ} 00^{\prime} S\right)$, región que está integrada en el Sistema de Corrientes Perú-Chile, la cual se caracteriza por presentar una elevada productividad biológica (Blanco et al. 2001), debido a intensos afloramientos costeros (surgencias) impulsados por vientos predominantes sur que se vuelven más intensos y persistentes durante el verano (Thomas et al. 1994; Escribano et al. 2004, Herrera \& Escribano 2006), los cuales en algunos puntos locales toman el carácter de permanentes (Fuenzalida 1992). La información analizada proviene de los desembarques que realiza la flota cerquera y que se extrae desde la costa hasta las 200 millas náuticas, y se encuentra bajo el monitoreo del programa de 'Seguimiento de Pesquería Pelágicas de la Zona Norte', desarrollado por el Instituto de Fomento Pesquero (IFOP) y financiado por la Subsecretaria de Pesca. El periodo de estudio consideró la serie 2000-2010 analizando como promedio semanal 2.000 ejemplares y 150 hembras, sobre la cual se realizó un análisis macro y microscópico, respectivamente. Se consideró el período de mayor actividad reproductiva (julio a diciembre con valores de IGS > 5), debido a que los cambios que tiene este indicador está directamente relacionado con la maduración y la liberación de gametos, los que a su vez dependen del tamaño corporal del ejemplar de (Cubillos $2005)^{1}$. No obstante, entre el 2001 y 2003 se realizó muestreo y análisis histológico sistemático durante todo el año.

\section{ANÁlisis MACro Y MiCroscóPICo}

El diseño de muestreo aplicado para los análisis macro y microscópico correspondió a un muestreo aleatorio simple bi-etápico (viaje, captura), donde los ejemplares fueron medidos en longitud $( \pm 0,5 \mathrm{~cm})$, peso total $( \pm 0,01 \mathrm{~g})$, peso eviscerado $(0,01 \mathrm{~g})$ y peso de la gónada $(0,01 \mathrm{~g})$. Para el análisis macroscópico cada gónada fue identificada mediante la aplicación de criterios macroscópicos siguiendo la escala de clasificación de desarrollo ovárico de Holden \& Raitt (1975). Para establecer la evolución de la actividad reproductiva se utilizan indicadores macroscópicos como el IGS y proporción de hembras maduras (fase III y V). El IGS es un indicador ampliamente utilizado que describe las variaciones en el peso de la gónada asociado al proceso de maduración, respecto al peso del pez (peso corporal menos el peso de la gónada) (De Vlaming et al. 1982, Erickson et al. 1985, West 1990). El índice se estima según la ecuación:

$$
\begin{gathered}
I \hat{G} S_{i}=\frac{W g_{i}}{W_{i}} 100 \\
I \hat{G} S=\sum_{i=1}^{n} I \hat{G} S_{i} / n \\
\hat{V}(I G S)=\frac{1}{n} \frac{\sum_{i=1}^{n}\left(I G S_{i}-I \hat{G} S\right)^{2}}{n-1}
\end{gathered}
$$

${ }^{1}$ Cubillos L. 2005. Biologia pesquera y evaluación de stock, 198 pp. Laboratorio de Evaluación de Pesquerías Marinas y Análisis de pesquerías, Departamento de Oceanografía, Universidad de Concepción, Concepción. 
donde, $W g$ corresponde al peso del ovario y $W$ al peso eviscerado del individuo y $\mathrm{n}$ corresponde al número total de ejemplares analizados.

Para el análisis microscópico la gónada de cada hembra fue fijada en una solución de formalina al 10\%, neutralizada con sodio Di-Hidrogeno fosfato puro $\left(\mathrm{Na}_{2} \mathrm{H}_{2} \mathrm{PO}_{4}\right)$ y $\mathrm{Di}$ Sodio Hidrogeno fosfato 12-Hidrato $\left(\mathrm{Na}_{2} \mathrm{HPO}_{4}\right.$. $\left.12 \mathrm{H}_{2} \mathrm{O}\right)$. Una porción de cada ovario se deshidrató y se incluyó en parafina, realizando cortes de $5 \mu \mathrm{m}$ de espesor, los cuales fueron teñidos con hematoxilina y contrastados con eosina. El diagnóstico histológico fue realizado de acuerdo al procedimiento descrito por Hunter \& Goldberg (1980) y Hunter \& Macewicz (1980, 1985), utilizando una escala específica reportada por Sepúlveda et al. (1999) orientado a identificar ovocitos en diferentes grados de desarrollo, folículos postovulatorios y estadios atrésicos. La clasificación de estadios de la degeneración de los folículos postovulatorios se realizó de acuerdo a señalado por Alday et al. (2008) y adecuada a E. ringens por Claramunt et al. (2007) quienes consideran 7 estadios para la anchoveta.

El monitoreo histológico de la actividad de desove requiere de la observación sistemática de la presencia de ejemplares con folículos postovulatorios (FPO) debido que a través de éstos es posible estimar la frecuencia del desove y de ovocitos hidratados en el ovario, para estimar la proporción de individuos que alcanzaron este estado en el stock parental, es decir, determinar el componente de la fracción adulta en actividad de desove inminente y reciente. Como indicadores histológicos de desove y de atresia ovocitaria se calcularon el índice de actividad de desove (IAD) (Hunter \& Macewicz 1985, Díaz et al. 2013²) y el índice de atresia ovárica (IAO). Como indicador de actividad ovárica se agruparon aquellas hembras con fases ováricas en desarrollo:

$$
I A D=\frac{N H D_{(0)}-N H D_{(-1)}}{\text { Número de hembras activas }} * 100
$$

$\mathrm{NHD}_{(0)}$ : Número de hembras que desovarán (hidratadas), más las que desovaron la noche de la captura, cuyos ovarios presentan ovocitos hidratados y FPO I.

$\mathrm{NHD}_{(-1)}$ : Número de hembras que desovaron la noche anterior de la captura, cuyos ovarios presentan FPO II al V.
Asimismo se determinó el índice de atresia ovárica (IAO) indicador inverso al IAD, el cual requiere conocer la incidencia de hembras con atresia ovocitaria masiva (> $50 \%$ ovocitos vitelados con atresia) (Hunter \& Macewicz 2003), según la siguiente expresión:

$$
I A O=\frac{\text { NHAO }}{\text { Número de hembras activas }} * 100
$$

NHAO: Número de hembras con atresia ovárica.

\section{Resultados}

\section{ANÁLISIS MACroscópico}

El IGS evidenció un ciclo anual similar entre el 2000 y 2010 , presentando a partir de enero una tendencia descendente para alcanzar un valor mínimo en abril (reposo reproductivo), para registrar un incremento paulatino en los valores a partir de mayo con IGS máximos hacia mediados y finales de invierno austral (agosto y septiembre), manteniendo altos valores de IGS hasta diciembre (Fig. 1a). La proporción de hembras maduras presentó un ciclo similar al IGS, con valores máximos de julio a diciembre (>90\%), decreciendo hasta llegar al mínimo en abril (Fig. 1b). En base a los indicadores macroscópicos de IGS y la proporción de hembras maduras es posible determinar el periodo de máxima actividad reproductiva que se establece entre julio y se extiende hasta diciembre, periodo que se caracteriza por valores de IGS superiores a $6 \%$ y proporción de hembras maduras mayor al 90\%, sin embargo se observó una baja relativa en los valores de IGS en octubre.

\section{ANÁLISIS MICroscóPICo}

Entre 2001 y 2003, en que se dispone de información anual, se observó de enero a mayo una alta variabilidad del IAD con valores que no superaron el $20 \%$ y niveles de IAO mayores al 6\% (Figs. 2 y 3a). A partir de junio el IAD presentó un incremento, concentrándose los índices más altos entre mediados de julio y octubre, con máximos que se manifiestan en el bimestre agosto-septiembre. De octubre a diciembre el IAD presenta una tendencia descendiente con índices similares al primer semestre del año. Entre el 2004 y 2010 el monitoreo se realizó de julio a diciembre, meses que cubren el período de mayor actividad

\footnotetext{
${ }^{2}$ Díaz E, MG Böhm, C Hernández \& C Bernal. 2013. Determinación del período reproductivo y máxima intensidad de desove (MID) de la anchoveta en la zona norte de Chile. Informe Coyuntural, Convenio asesoría integral para la toma de decisiones en pesca y acuicultura, 2013: actividad 1: recursos pelágicos pesquería pelágica zona norte. Subsecretaria de Economía, Santiago de Chile, pp. 1-20.
} 
a)

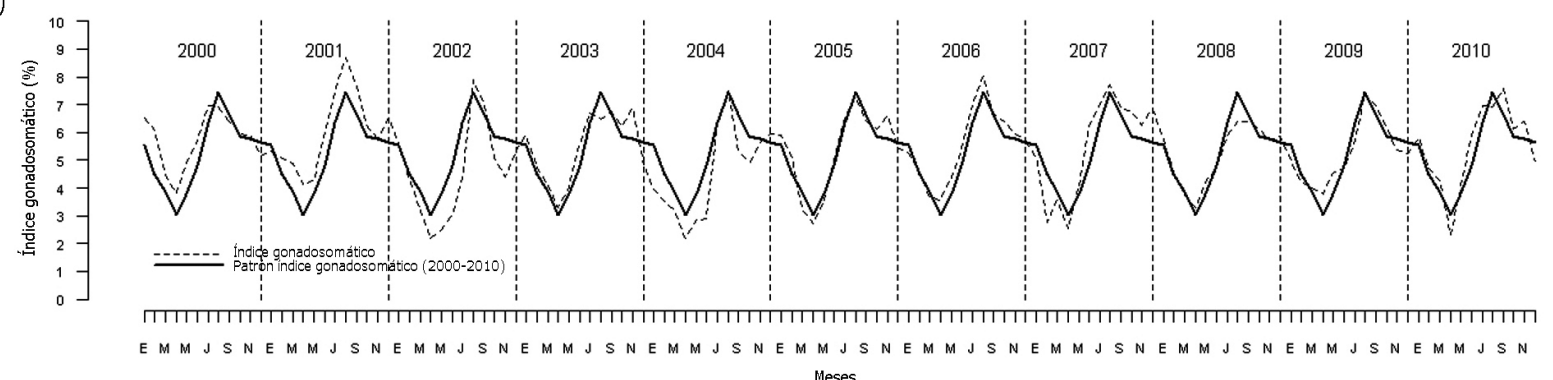

b)

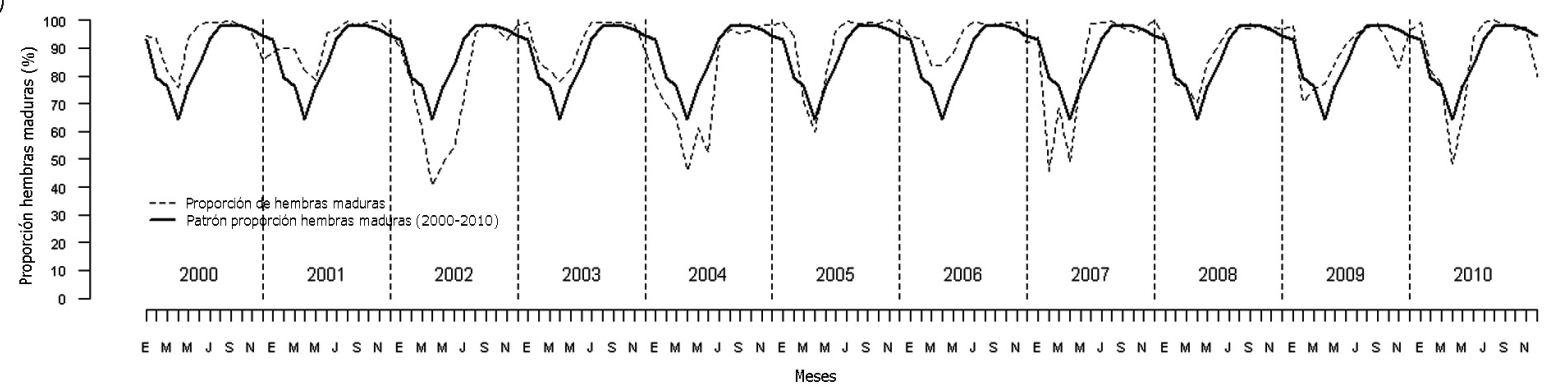

Figura 1. Series históricas mensuales de la variación de anchoveta en la zona norte de Chile. Años 2000-2010 a) índice gonadosomático (IGS) y b) proporción de hembras maduras (Fase maduración $\mathbf{3}$ y 4) / Historical monthly series of the variation of anchoveta of northern Chile. Years 2000-2010 a) gonadosomatic index (IGS) and b) proportion of mature females (Phase ripeness 3 and 4 )

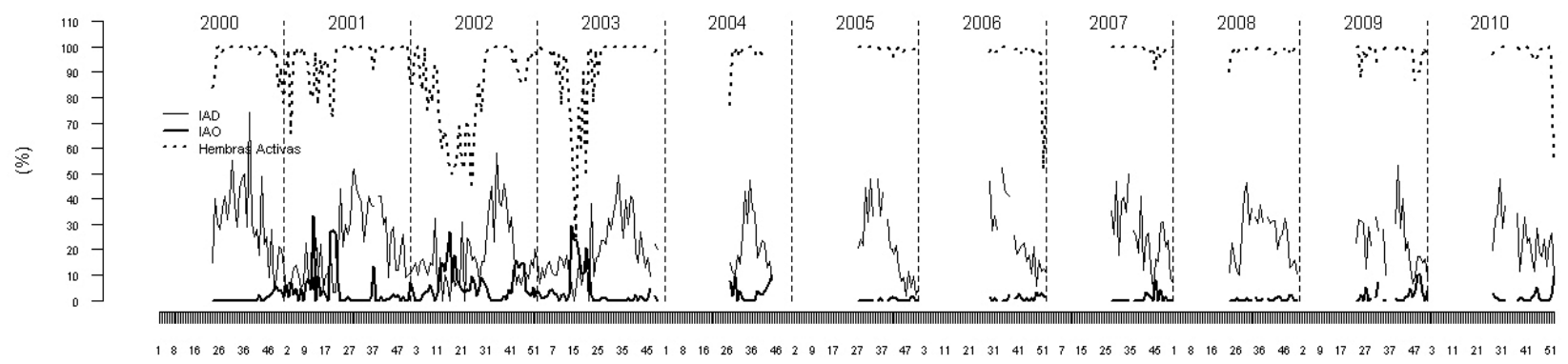

Semanas

Figura 2. Variación semanal de índices microscópicos, índice de actividad de desove (IAD), índice de atresia ovárica (IAO) y hembras activas para la zona norte de Chile. 2000-2010 / Weekly variation of microscopic indexes, index of activity of spawning (IAD), ovarian atresia index (IAO) and active females of northern Chile. 2000-2010

reproductiva. Al respecto, en las primeras semanas de julio se observa una alta variabilidad de los valores de IAD, luego a partir la segunda quincena de julio el IAD se incrementa con valores promedio igual o superior a $25 \%$ y valores de IAO cercanos a cero. Desde octubre en adelante se registró una baja del IAD menor a $25 \%$ y un aumento gradual del IAO .

\section{Discusión}

Los indicadores histológicos de IAD e IAO resultaron una herramienta útil para la determinación del período de máxima intensidad de desove (MID) siendo sus ventajas principales 1) el uso en tiempo real validando los indicadores macroscópicos, 2) definir con mayor precisión el inicio y fin del proceso de desove y 3) análisis más exhaustivo de la variabilidad interanual del inicio y fin del MID. En este sentido, los resultados registran el período de máxima intensidad de desove entre mediados de julio y mediados de octubre, estableciendo como criterio experto basado en indicadores macro y microscópico con valores de $\mathrm{IGS} \geq 6 \%$ e IAD $\geq 25 \%$ manteniendo estos 

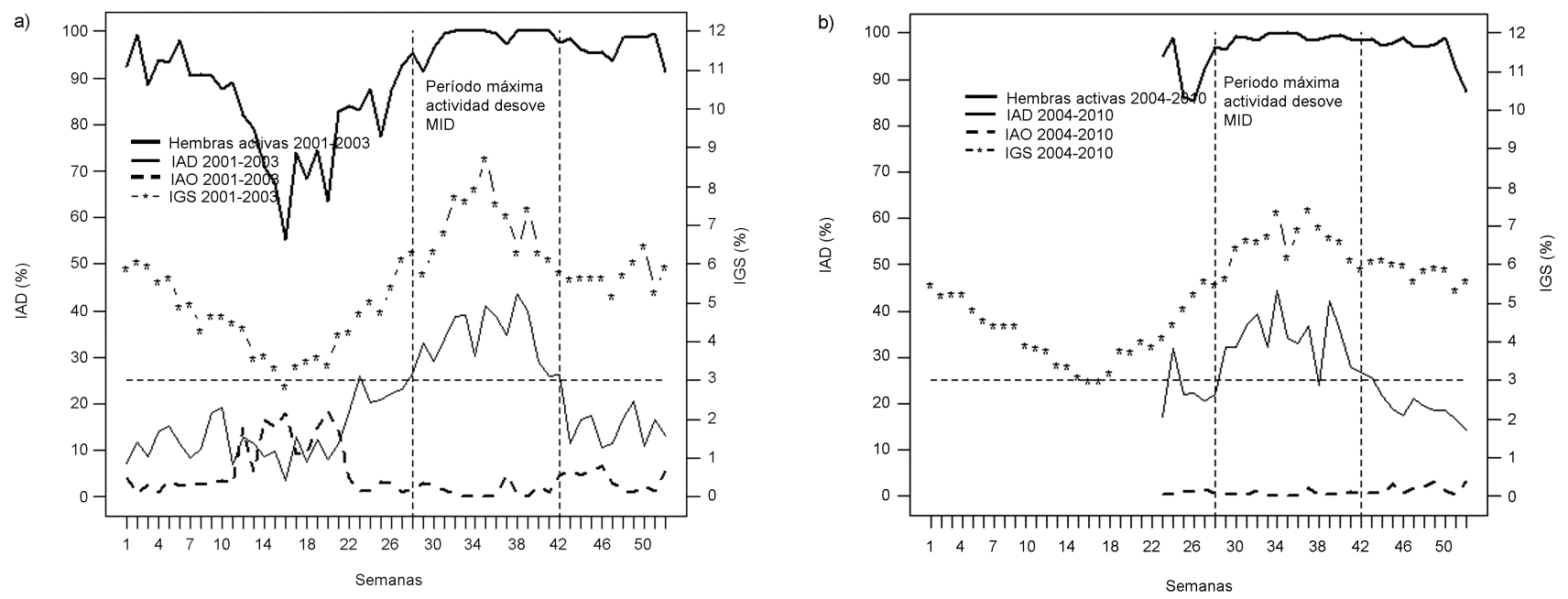

Figura 3. Variación semanal promedio del índice gonadosomático (IGS), índices de actividad de desove (IAD), atresia ovárica (IAO) y proporción de hembras activas en la zona norte de Chile. (a) Periodo 2001-2003 y (b) Periodo 2004-2010. La línea punteada horizontal índica IAD=25\% y las verticales, el período máxima actividad de desove / Weekly average variation of the gonadosomatic index (IGS), activity of spawning index (IAD), ovarian atresia (IAO) and proportion of active females of northern Chile. (a) 2001-2003 period and (b) 2004-2010 period. The dotted horizontal line indicates $I A D=25 \%$ and the vertical ones, the period maximum activity of spawning

niveles durante 2 semanas no necesariamente consecutivas lo que gatilla el inicio de la máxima intensidad de desove (Díaz et al. 2013). Sin embargo, existe una variabilidad interanual en el inicio y fin del proceso, registrando un desfase en el 2002 y 2004, detectándose a inicios de agosto. Por otra parte, el fin del proceso también presentó un cambio, adelantándose (caso del 2003 y 2010) o retrasándose, como lo que ocurrió en el 2009.

El desfase tanto en el inicio como el fin del proceso de la serie analizada del MID, es de aproximadamente una semana (excepto 2004). Esta baja variabilidad en el inicio y fin del proceso podría ser considerada una ventaja para la administración de la anchoveta, dado el alto costo que representa la histología es posible seguir aplicando esta metodología en los períodos de máxima actividad reproductiva.

Por otro lado, la duración del proceso de máxima actividad de desove se puede establecer en aproximadamente 3,5 meses, no obstante el 2004 se registró una reducción a menos de 2 meses. Tanto los desfases al inicio y fin del proceso como la duración de éste podrían ser explicados por cambios en la condición ambiental de la zona de estudio. Al respecto, Yáñez et al. (2008), señalan que la intrusión de aguas sub-tropicales modifican las condiciones ambientales, que afectan el comportamiento de anchoveta, asimismo existen antecedentes que indican que la estacionalidad reproductiva puede ser influenciada por factores exógenos principalmente por temperatura, ubicación geográfica (latitud), tamaño y edad de la población (Castro et al. 2001, Basilone et al. 2006). En este contexto la evolución mensual de los índices macroscópicos evidenció una mayor actividad reproductiva a partir de julio alcanzando un máximo en agosto y altos índices que se mantuvieron hasta diciembre. Estos resultados concuerdan con lo mencionado por Martínez (1990), quiénes señalaron que la máxima actividad reproductiva poblacional del recurso para la zona norte de Chile, ocurre entre agosto y noviembre, tendencias similares a 10 reportado para la anchoveta de la zona centro norte y centro sur de Chile (Cubillos 1991, Cubillos et al. 1999, Leal et al. 2009).

El análisis microscópico confirmó la condición de la anchoveta como desovador parcial (Alheit et al. 1984, Santander et al. 1984) y mediante el análisis de los datos de la serie histórica (2000-2010), se ha observado que el periodo de máxima actividad de desove presenta una periodicidad en el ciclo reproductivo relacionado con las condiciones ambientales y estacionales. Tal comportamiento puede ser explicado por el ciclo de vida de la anchoveta y su distribución a lo largo de la corriente de Humboldt el que incluye una serie de eventos secuenciales (reproducción en invierno, crecimiento de juveniles en primavera y a principios de verano, acumulación de lípidos a finales del verano y principios de otoño). Asimismo, se manejan 3 hipótesis no independientes que pueden 
explicar la sincronización de la estrategia reproductiva en invierno, 1) El desove en invierno facilita la retención de huevos y larvas, como resultado de que los vientos norte inducen el transporte de huevos y larvas hacia la costa (Castro et al. 2000, 2001), 2) el desove en invierno puede ser beneficioso para larvas y juveniles cuando alcanzan una alta tasa de crecimiento (Cubillos et al. 2001), ya que les permite aprovechar la alta producción de plancton que hay en primavera y a inicios del verano y 3 ) el desove en invierno puede ser favorecido como resultado de las reservas acumuladas a finales del verano para la reproducción en invierno (Hunter \& Leong 1981). Todas estas hipótesis incluyen diferentes procesos en la historia de vida de los peces (Castro et al. 2009).

\section{Agradecimientos}

Los autores agradecen a los observadores científicos (OC) del Instituto de Fomento Pesquero (IFOP) por el monitoreo de los desembarques realizado en tierra y a bordo de naves pesqueras en la zona norte de Chile, así como también a los profesionales y técnicos del laboratorio de histología de Iquique.

\section{LITERATURA CITADA}

Alday A, A Uriarte, M Santos, I Martín, A Martínez \& L Motos. 2008. Degeneration of postovulatory follicles of the Bay of Biscay anchovy (Engraulis encrasicolus L.). Scientia Marina 72(3): 565-575.

Alheit J, VH Alarcón \& BJ Macewics. 1984. Spawning frequency and sex ratio in the Peruvian anchovy, Engraulis ringens. CALCOFI Reports 25: 43-52.

Arancibia H, L Cubillos, J Remmaggi \& R Alarcón. 1994. Determinación de la talla de primera madurez sexual y fecundidad parcial en la sardina común, Strangomera bentincki (Norman, 1936), del área de Talcahuano, Chile. Biología Pesquera 23: 11-17.

Basilone G, C Guisande, B Patti, S Mazzola, A Cuttitta, A Bonanno, AR Vergara \& I Maneiro. 2006. Effect of habitat conditions on reproduction of the European anchovy (Engraulis encrasicolus) in the Strait of Sicily. Fisheries Oceanography 15(4): 271-280.

Blanco JL, AC Thomas, ME Carr \& PT Strub. 2001. Seasonal climatology of hydrographic conditions in the upwelling region off northern Chile. Journal of Geophysical Research 106: 11451-11467.

Buitrón B \& A Perea. 2000. Aspectos reproductivos de la anchoveta peruana durante el periodo 1992-2000. Boletín, Instituto del Mar de Perú 19: 45-54.

Castro LR, G Salinas \& EH Hernández. 2000. Environmental influences on winter spawning of the anchoveta, Engraulis ringens, off Central Chile. Marine Ecology Progress Series 197: $247-258$.
Castro L, A Llanos, J Blanco, E Tarifeño, R Escribano \& M Landaeta. 2001. Influence of latitudinal variations in spawning habitat characteristics on the early life history traits of the anchoveta, Engraulis ringens, off northern and central Chile. GLOBEC Report 16: 42-45.

Castro LR, G Claramunt, MC Krautz, A Llanos-Rivera \& P Moreno. 2009. Egg trait variation in anchoveta Engraulis ringens: a maternal response to changing environmental conditions in contrasting spawning habitats. Marine Ecology Progress Series 381: 237-248.

Chávez F, M Ñiquen, J Csirke, A Bertrand, C Wostnitza \& R Guevara-Carrasco. 2009. Fifth international panel on the anchoveta towards an ecosystem approach to fisheries. GLOBEC International Newsletter 15: 14-15.

Claramunt G, G Herrera, P Pizarro, J Pizarro, R Escribano, M Oliva, A Olivares \& A Zuleta. 1997. Evaluación del stock desovante de anchoveta por el método de producción de huevos en la I y II regiones. Fondo de Investigación Pesquera, Universidad Arturo Prat, Iquique. Informe Final, Proyecto FIP 96-01: 1-75. <http://www.fip.cl/FIP/ Archivos/pdf/informes/IT\%2096-01.pdf>.

Claramunt G, R Serra, LR Castro \& L Cubillos. 2007. Is the spawning frequency dependent on female size? Empirical evidence in Sardinops sagax and Engraulis ringens off northern Chile. Fisheries Research 85: 248257.

Csirke J, R Guevara-Carrasco, G Cárdenas, M Ñiquen \& A Chipollini. 1996. Situación de los recursos anchoveta (Engraulis ringens) y sardina (Sardinops sagax) a principios de 1994 y perspectivas para la pesca en el Perú, con particular referencia a las regiones norte y centro de la costa peruana. Boletín, Instituto del Mar de Perú 15: 1-23.

Cubillos L. 1991. Estimación mensual de la biomasa, reclutamiento y mortalidad por pesca de la anchoveta (Engraulis ringens) de la zona norte de Chile en el período 1986-1989. Biología Pesquera 20: 49-59.

Cubillos L \& G Claramunt. 2009. Length-structured analysis of the reproductive season of anchovy and common sardine off central southern Chile. Marine Biology 156: 1673-1680.

Cubillos LA, DF Arcos, M Canales \& D Bucarey. 2001. Seasonal growth of small pelagic fish off Talcahuano (37S$73 \mathrm{~W})$, Chile. A consequence of their reproductive strategy to seasonal upwelling? Aquatic Living Resources 14: 115124.

Cubillos L, TM Canales, D Bucarey, A Rojas \& R Alarcón. 1999. Época reproductiva y talla media de primera madurez de Strangomera bentincki, y Engraulis ringens en el período 1993-1997 en la zona centro sur de Chile. Investigaciones Marinas 27: 73-85.

De Vlaming V, G Grossman \& F Chapman. 1982. On the use of the gonadosomatic index. Comparative Biochemical and Physiology 73A: 31-39.

Einarsson H, B Rojas de Mendiola \& H Santander. 1966. Los desoves de peces en aguas peruanas durante 19611964. Memoria del Primer Seminario Latinoamericano del Océano Pacífico Oriental, Universidad Nacional Mayor de San Marcos Lima, Perú, pp. 110-127. 
Erickson DL, MJ Harris \& GD Grossman. 1985. Ovarian cycling of tilefish, Lopholatilus chamaeleonticeps Goode and Bean, from the South Atlantic Bight, U.S.A. Journal of Fish Biology 27: 1131-1146.

Escribano R, G Daneri, L Farías, V Gallardo, H González, D Gutiérrez, C Lange, C Morales, O Pizarro, O Ulloa \& M Braun. 2004. Biological and chemical consequences of the 1997-1998 El Niño in the Chilean coastal upwelling system: a synthesis. Deep-Sea Research Part II, 51: 23892411.

Fuenzalida R. 1992. Proceso de surgencia en la región norte de Chile, latitudes $20^{\circ} 30^{\prime} \mathrm{S}-21^{\circ} 45^{\prime} \mathrm{S}$ ). Revista de Investigaciones Científicas y Tecnológicas, Serie Ciencias del Mar 2: 79-104.

Herrera L \& R Escribano. 2006. Factors structuring the phytoplankton community in the upwelling site off El Loa River in northern Chile. Journal of Marine Systems 61:1338.

Holden M \& D Raitt. 1975. Manual de ciencia pesquera. Parte 2. Métodos para investigar los recursos y su aplicación. FAO Documento Técnico de Pesca 115: 1-211.

Hunter JR \& S Goldberg. 1980. Spawning incidence and batch fecundity in the northern anchovy, Engraulis mordax. Fishery Bulletin 77(3): 641-652.

Hunter JR \& R Leong. 1981. The spawning energetics of female Northern anchovy, Engraulis mordax. Fishery Bulletin 79: 215-230.

Hunter JR \& B Macewicz. 1980. Sexual maturity, batch fecundity, spawning frequency and temporal pattern in the northern anchovy, Engraulis mordax, during the 1979 spawning season. CALCOFI Reports 21: 139-149.

Hunter JR \& B Macewicz. 1985. Measurement of spawning frequency in multiple spawning fishes. In: Lasker R (ed). An egg production method for estimating spawning biomass of pelagic fish: Application to the northern anchovy, Engraulis mordax. NOAA Technical Report, NMFS 36: 79-94.

Hunter JR \& B Macewicz. 2003. Improving the accuracy and precision of reproductive information used in fisheries. In: Kjesbu S, JR Hunter \& PR Witthames (eds). Modern approaches to assess maturity and fecundity of warm- and cold-water fish and squids. Fisken og Havet 12: 57-68.

Jordan R. 1976. Biología de la anchoveta. Parte I: Resumen del conocimiento actual. FAO Fisheries Technical Reports 185: 359-399.
Leal EM, LR Castro \& G Claramunt. 2009. Variability in oocyte size and batch fecundity in anchoveta (Engraulis ringens, Jenyns 1842) from two spawning areas off the Chilean coast. Scientia Marina 73: 59-66.

Martínez C. 1990. Diagnóstico de las principales pesquerías nacionales. En: Caballero L, G Böhm, R Gili, V Bocic, H Muñoz, P Barría, R Serra, H Hidalgo, P Sáez \& V Ortiz (eds). Estado de la situación y perspectivas del recurso. Pesquerías pelágicas Zona Norte (I-IV Región). Corporación de Fomento de la Producción (CORFO), e Instituto de Fomento Pesquero (IFOP), 1991(8): 1-119.

Mori J, B Buitrón, A Perea, C Peña \& C Espinoza. 2011. Interannual variability of the reproductive strategy of the Peruvian anchovy off northern-central Peru. Ciencias Marinas 37(4B): 513-523.

Purca S, M Graco, D Gutiérrez, B Dewitte, J Tam, A Bertrand, F Chávez, R Flores, J Ledesma, L Vásquez, M Messié, K Goubanova, O Morón, C Nakazaki, S Peraltilla \& S Sánchez. 2010. Relación entre anchoveta y ambiente a diferentes escalas temporales. Boletín, Instituto del Mar del Perú 25: 13-21.

Santander H, J Alheit \& PE Smith. 1984. Estimación de la biomasa desovante de anchoveta peruana, Engraulis ringens, en 1981 por aplicación del Método de Producción de Huevos. Boletín, Instituto del Mar del Perú, Callao 8: 209250

Sepúlveda A, L Cubillos, S Núñez, T Canales, D Bucarey, A Rojas, J Oliva, P Barría, E Díaz, V Baros \& H Miranda. 1999. Antecedentes biológicos del stock desovante de anchoveta y sardina común de la V a IX regiones. Informe Final FIP 97-04: 1-191 <http://www.fip.cl/Archivos/Hitos/ Informes/inffinal\%2097-04.pdf>

Thomas AC, F Huang, PT Strub \& C James. 1994. A comparison of the seasonal and interannual variability of phytoplankton pigment concentrations in the Peru and California Current Systems. Journal of Geophysical Research 99: 7355-7370.

West G. 1990. Methods of assessing ovarian development in fishes-a review. Australian Journal of Marine and Freshwater Research 41: 199-222.

Yáñez E, S Hormazábal, C Silva, A Montecinos, MA Barbieri, A Valdenegro, A Ordenes \& F Gómez. 2008. Coupling between the environment and the pelagic resources exploited off North Chile: ecosystem indicators and a conceptual model. Latín American Journal of Aquatic Research 36(2): 159-181. 